\title{
Dislocated Double-Layer Metal Gratings: An Efficient Unidirectional Coupler
}

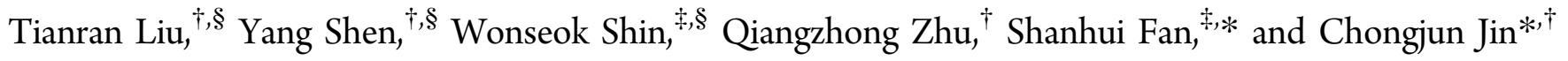 \\ ${ }^{\dagger}$ State Key Laboratory of Optoelectronic Materials and Technologies, School of Physics and Engineering, Sun Yat-sen University, \\ Guangzhou 510275, China \\ ${ }^{\ddagger}$ Department of Electrical Engineering, Ginzton Laboratory, Stanford University, Stanford, California 94305, United States
}

Supporting Information

\begin{abstract}
We propose theoretically and demonstrate experimentally a dislocated double-layer metal grating structure, which operates as a unidirectional coupler capable of launching surface plasmon polaritons in a desired direction under normal illumination. The structure consists of a slanted dielectric grating sandwiched between two gold gratings. The upper gold grating has a nonzero lateral relative displacement with respect to the lower one. Numerical simulations show that a grating structure with 7 periods can convert $49 \%$ of normally incident light into surface plasmons with a contrast ratio of 78 between the powers of the surface plasmons launched in two opposite directions. We explain the unidirectional coupling phenomenon by the dislocation-induced interference of the diffracted waves from the upper and lower gold gratings. Furthermore, we developed a simple and cost-effective technique to fabricate the structure via tilted two-

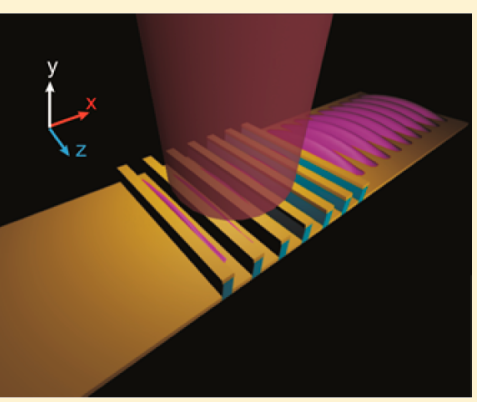
beam interference lithography and subsequent shadow deposition of gold. The experimental results demonstrate a coupling efficiency of $36 \%$ and a contrast ratio of 43 . The relatively simple periodic nature of our structure lends itself to large-scale lowcost fabrication and simple theoretical analysis. Also, unlike the previous unidirectional couplers based on aperiodic structures, the design parameters of our unidirectional coupler can be determined analytically. Therefore, this structure can be an important component for surface-plasmon-based nanophotonic circuits by providing an efficient interface between free-space and surface plasmon waves.
\end{abstract}

KEYWORDS: Unidirectional coupler, surface plasmon polariton, metal grating, Wood's anomaly

$\mathrm{M}$ odern information processing demands ultracompact photonic devices at the chip scale. Surface plasmon polaritons (SPPs) present an intriguing approach to implement ultracompact photonic devices, because with SPPs one may overcome the diffraction limit encountered in conventional optics. ${ }^{1,2}$ Many photonic devices based on SPPs have been investigated in recent years ${ }^{3,4}$ and integrated plasmonic circuits are starting to emerge.

To envision the incorporation of such plasmonic circuits into an overall optical system, however, an important challenge is to be able to generate SPPs efficiently from freely propagating light and control the direction in which they are launched. Standard grating structures can be used to launch SPPs from free space beam, but for normally incident light this typically results in simultaneous launching of SPPs in opposite directions. ${ }^{5}$ There have been unidirectional SPP excitation achieved by illuminating grating couplers with obliquely incident beams ${ }^{6-8}$ or circularly polarized beams. ${ }^{9,10}$ However, the coupling from linearly polarized beams at normal incidence is more desirable in optical systems.

There have been several approaches to achieve unidirectional excitation of SPPs from linearly polarized beams at normal incidence. As a first approach, one could use an individual subwavelength element, such as a slit, groove, or a plasmonic resonant cavity, to excite SPP from normally incident beams.
The unidirectionality can then be accomplished by placing asymmetric structures around a single slit ${ }^{11-19}$ or by achieving direction-dependent constructive or destructive interference between asymmetric two slits ${ }^{20}$ and grooves, ${ }^{21}$ as well as cavities with different resonant frequencies. ${ }^{22,23}$ However, the coupling efficiency of such a construction is fundamentally limited by the electromagnetic cross section of the subwavelength elements used. Because these elements typically have an electromagnetic cross section smaller than the wavelength of light, ${ }^{24}$ the coupling efficiency is inherently low for a free-space incident beam with a typical cross-sectional dimension of at least a few wavelengths. As a result, only a small portion of the incident light can be coupled into SPPs, while most of the incident light is reflected.

To achieve high-efficiency unidirectional excitation of SPP from free-space beams one should consider specially designed asymmetric grating structures, which have physical dimensions on the order of several wavelengths for better mode-matching with the incident beam. Periodic structures based on slanted gratings $^{25,26}$ and asymmetrical double-layer metal gratings without vertical spacing ${ }^{27}$ have been proposed but the realized

Received: March 18, 2014

Revised: June 5, 2014

Published: June 13, 2014 
asymmetrical coupling effect was weak due to the limitations in experiment. ${ }^{26}$ An alternative method is to use limited aperiodic groove arrays with modulated period, ${ }^{28}$ width, ${ }^{29-31}$ or both width and depth of grooves. ${ }^{32}$ These grooves have to be etched by focused ion beam (FIB) because each of these grooves has unique parameters. Also, due to the nature of aperiodicity one needs to specifically design and optimize the structure for different operating wavelengths.

In this Letter, we theoretically propose and experimentally demonstrate dislocated double-layer metal gratings that serve as efficient unidirectional couplers for SPP. The structure is composed of a slanted dielectric grating sandwiched between two gold gratings (Figure 1). The upper gold grating has a

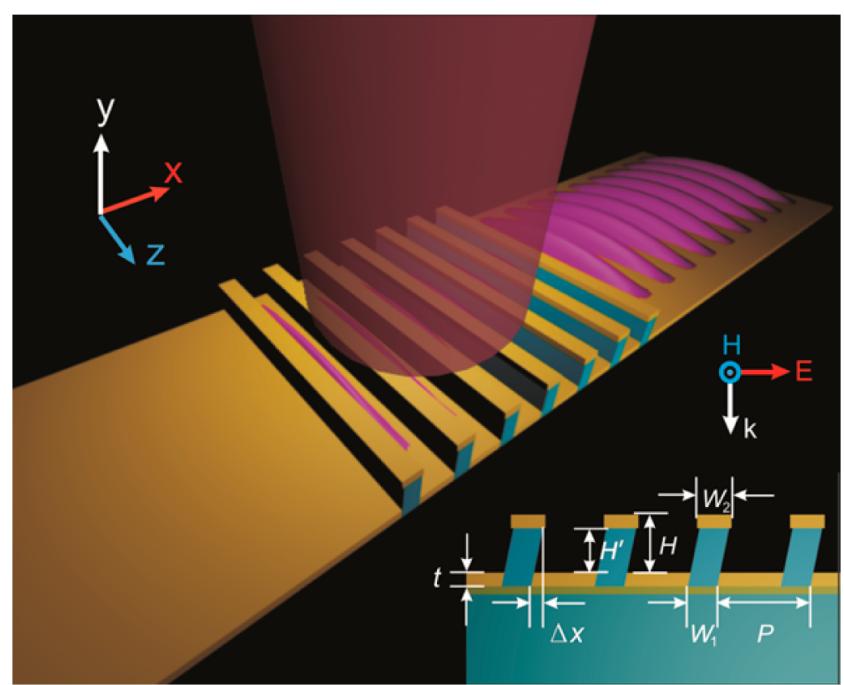

Figure 1. Schematic of launching unidirectional SPP from normally incident beam by a dislocated double-layer metal grating. (Inset) The geometric parameters of the structure. The period and thickness of the gold layers are fixed at $P=770 \mathrm{~nm}$ and $t=120 \mathrm{~nm}$ throughout this paper. $H$ and $H^{\prime}$ represent the thickness of the photoresist and spacer between the two metal gratings. The electric field of the incident waves is perpendicular to the gold grating stripes.

nonzero lateral relative displacement with respect to the lower one. We refer to such displacement as "dislocation" in this paper, and hence the entire structure as a "dislocated" doublelayer metal grating. The structure is simpler and easier to fabricate than most of previous designs. We show both numerically and experimentally that this structure provides high-efficiency high-contrast unidirectional launching of SPP from free-space beams. For this structure, we have developed a low-cost, simple, and scalable nanofabrication method consisting of tilted two-beam interference lithography and subsequent shadow deposition of gold. This fabrication method is also compatible with the modern silicon technology. Finally, we have developed a simple analytic model that accounts for the unidirectional effect in this system with which the unidirectional coupler for SPP can be designed analytically.

Figure 1 schematically shows the configuration of a dislocated double-layer metal grating. A slanted dielectric grating is sandwiched between two metal gratings. Double-layer metal gratings have been previously explored. ${ }^{33-42}$ Here we found that introducing the dislocation between upper and lower metal gratings results in a direction-dependent interference effect, which can be exploited to launch the SPP unidirectionally. The operating wavelength of the unidirectional coupler is determined by the period and thickness of photoresist. At resonance, the dislocated double-layer metal grating strongly interacts with light, and the waves diffracted by the upper and lower metal gratings constructively or destructively interfere with each other, depending on the direction and degree of the dislocation of the two metal gratings. With proper design, the waves propagating to one direction can be canceled out completely by destructive interference, resulting in a unidirectional coupling for SPP.

We perform numerical simulation using finite-difference time-domain (FDTD) method (FDTD solutions, Lumerical). The period of gratings $(P)$ and thickness $(t)$ of both gold gratings are 770 and $120 \mathrm{~nm}$, respectively. $H$ and $\Delta x$ refer to the thickness of photoresist gratings and dislocation between two gold gratings, respectively. $W_{1}$ and $W_{2}$ represent the widths of the photoresist stripes and upper grating metal stripes, respectively. The dielectric permittivity of gold is taken from the tabulated data. ${ }^{43}$ The refractive index of the photoresist and substrate are 1.61 and 1.46 , respectively. The incident light is TM polarized as indicated in the inset of Figure 1.

To demonstrate that a dislocated double-layer metal grating functions as a unidirectional coupler for SPP, we consider a finite-size coupler connected with gold film at both the left and right sides and illuminate the coupler with a Gaussian beam. The parameters of the grating are $W_{1}=230 \mathrm{~nm}, W_{2}=270 \mathrm{~nm}$, $H=530 \mathrm{~nm}, \Delta x=140 \mathrm{~nm}, P=770 \mathrm{~nm}$, and $t=120 \mathrm{~nm}$. Figure 2a shows the simulated electric field amplitude distribution of a grating with 7 periods, normally illuminated by a Gaussian beam with a waist diameter $(D)$ of $4000 \mathrm{~nm}$ and wavelength of $895 \mathrm{~nm}$. The incident wave, through the coupling to the grating, can be guided in the dislocated doublelayer metal grating and turn into an SPP wave that propagates only to the right.

Figure $2 \mathrm{~b}$ shows that the effect of the grating width on the coupling efficiency and the contrast ratio $E_{\mathrm{R}}$. The coupling efficiency is defined as the power of SPPs to the desired direction divided by the power of source, and the contrast ratio $E_{\mathrm{R}}$ is defined as the ratio between the power of launched SPPs to the desired direction and that to the opposite direction. The coupling efficiency for SPPs peaks when the width of the grating is about 1.4 times of the beam waist diameter (which corresponds to approximately 7 grating periods for the beam waist diameter $D=4000 \mathrm{~nm}$ ). Further increase of grating width decreases the coupling efficiency. The existence of a peak in coupling efficiency with respect to $L / D$ is due to the two opposite effects in this coupling process: light coupled into the grating structure increases with larger grating width but more power is either absorbed by these gold stripes or rescattered back to free space as grating width increases. The optimized value of grating width depends on the balance between these two effects. On the other hand, the contrast ratio increases nearly linearly with $L / D$. The $E_{\mathrm{R}}$ could be around 250 when the grating width is three times the beam waist diameter.

For a grating with a given period $N$, the diameter of the beam waist has opposite influences on the contrast ratio and coupling efficiency. Figure $2 \mathrm{c}$ illustrates this for the grating with $N=7$. Larger diameter results in higher coupling efficiency but lower contrast ratio because the part of light incident to the left edge of the structure could directly couple to the SPP propagating to the left side without further interference.

As a conclusion, the grating structure here has sufficient degree of freedom to enable optimization for either the coupling efficiency or contrast ratio. A contrast ratio as high as 


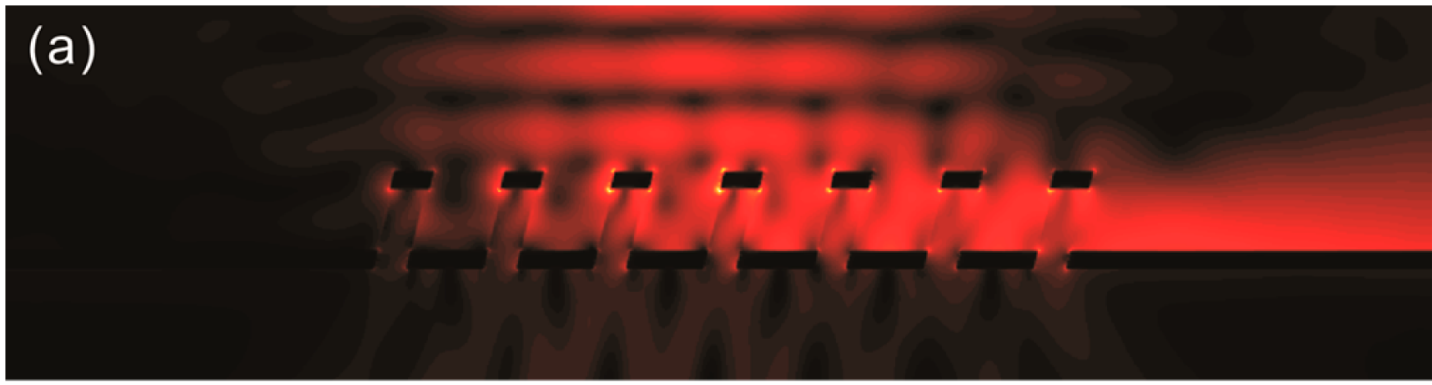

(b)

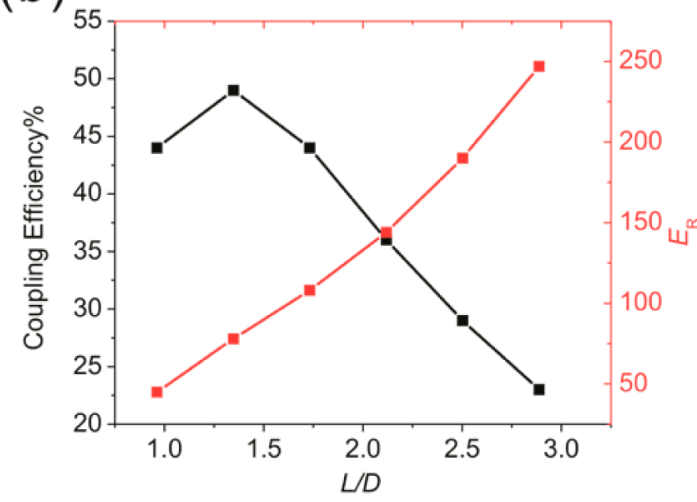

(c)

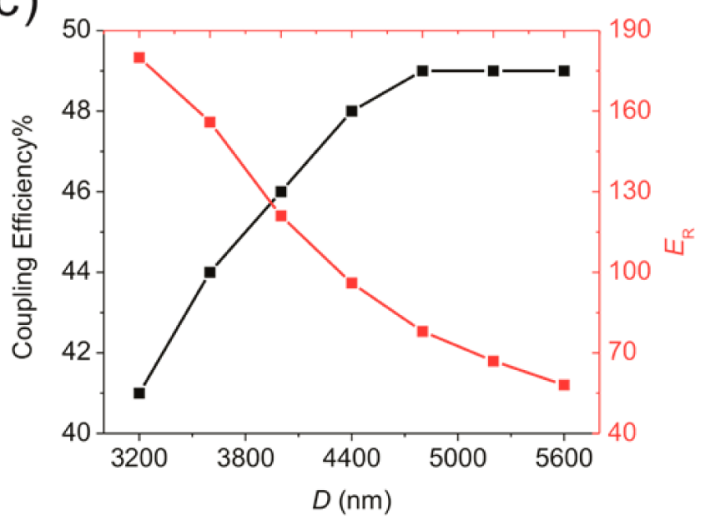

Figure 2. (a) The simulated electric field amplitude distribution of a dislocated double-layer metal grating with 7 periods illuminated by a Gaussian beam with a waist diameter of $4000 \mathrm{~nm}$ and wavelength of $895 \mathrm{~nm}$. (b) The effect of the grating width $L$ (normalized by the beam waist diameter $D$ $=4000 \mathrm{~nm}$ ) on the unidirectional coupling efficiency and contrast ratio $E_{\mathrm{R}}$ at the wavelength of $917 \mathrm{~nm}$. (c) The effect of the beam waist diameter $D$ of the incident Gaussian beam on the unidirectional coupling efficiency and contrast ratio $E_{\mathrm{R}}$ for the structure with 7 periods at the wavelength of $917 \mathrm{~nm}$. The other parameters of the structure are $W_{1}=230 \mathrm{~nm}, W_{2}=270 \mathrm{~nm}, H=530 \mathrm{~nm}$, and $\Delta x=140 \mathrm{~nm}$.
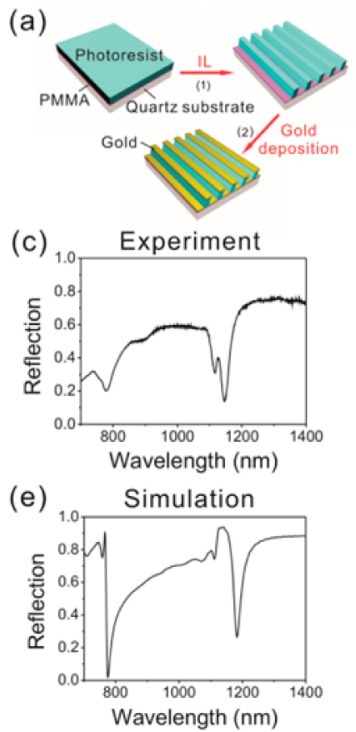

(b)

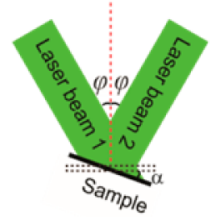

(d)
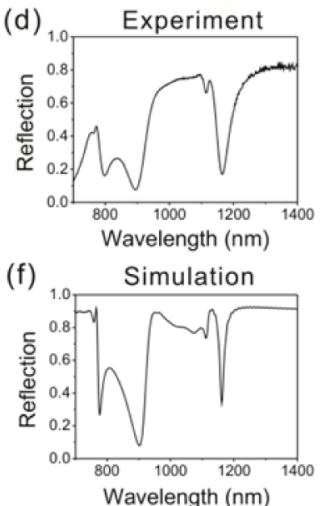
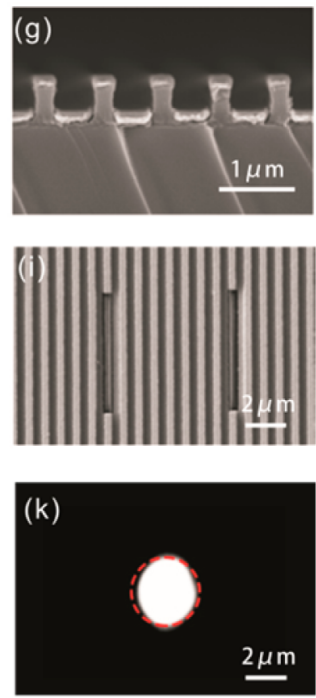
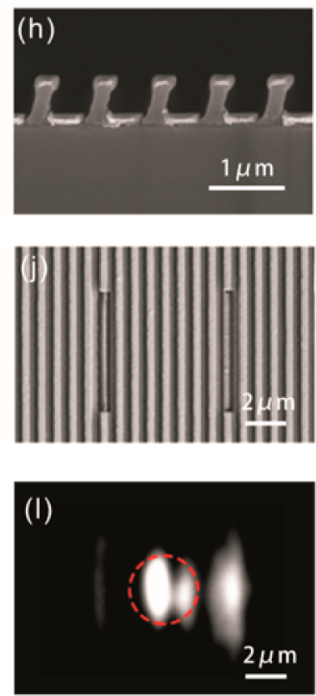

Figure 3. (a) The fabrication procedure for a dislocated double-layer metal grating, where IL refers to interference lithography. (b) Interference lithography geometry for preparing the slanted photoresist grating. $\varphi$ is the incident angle of two laser beams with respect to the normal (red dashed line); $\alpha$ is the rotation angle with respect to the plane that is perpendicular to the angle bisector formed by these two laser beams. (c,d) The measured reflection spectra of the nondislocated structure and dislocated structure, respectively. (e,f) The simulated reflection spectra of the nondislocated structure and dislocated structure, respectively. $(\mathrm{g}, \mathrm{h})$ The cross-sectional views of SEM images for the nondislocated structure and dislocated structure. $(\mathrm{i}, \mathrm{j})$ The top views of the nondislocated structure and dislocated structure with 7 periods and large grooves at both sides. (k,l) The microscope photos of the nondislocated structure (in (i) and dislocated structure (in j) illuminated by a focused Gaussian beam with a wavelength of $895 \mathrm{~nm}$ and pulse width of around $120 \mathrm{fs}$. The red dashed-circle represents the incident laser spot. The fabricated structure deviates slightly from the optimal design due to limited experimental precision. The actual parameters of sample are $H=490 \mathrm{~nm}, W_{1}=240 \mathrm{~nm}, W_{2}=290$ $\mathrm{nm}, H=770 \mathrm{~nm}, t=120 \mathrm{~nm}$, and $\Delta x=140 \mathrm{~nm}$ (for dislocated structure). 
78 can be reached with a considerable coupling efficiency of $49 \%$ with $N=7$ for a beam with a waist diameter of $4000 \mathrm{~nm}$. For a beam with a waist diameter of $3200 \mathrm{~nm}$, the contrast ratio can reach 180, and the coupling efficiency is still over $41 \%$. The coupling loss comes from two aspects: (i) the structure is not fully periodic due to a limited size, thus the grating coupling is not perfect, and (ii) a portion of light is dissipated by metal gratings before it turns into the unidirectional SPPs. Therefore, the size of the dislocated double-layer metal grating and diameter of the incident beam waist can influence the coupling performance for SPPs.

The overall performance of our design as a unidirectional coupler for SPPs is comparable to some of the best design in the literature. ${ }^{26,29}$ Importantly, unlike some of the aperiodic designs previously published, the simple periodic geometry of our design lends itself very well to large-scale low-cost fabrication techniques. To realize our design, we developed a nanofabrication method, which combines tilted interference lithography with shadow deposition of gold. The procedure is schematically illustrated in Figure 3a. We first prepared a slanted photoresist grating by rotating the sample at an angle $\alpha$ with respect to the plane that is perpendicular to the angle bisector formed by two laser beams during exposure (Figure $3 \mathrm{~b})$. A shadow deposition of gold was then employed. The lateral dislocation $\Delta x$ can be varied by adjusting the rotation angle $\alpha$. The periodicity of the grating is determined by the incident angle of the laser beams $\varphi$ and the rotation angle $\alpha$. A poly(methyl methacrylate) (PMMA) layer at a thickness of $\sim 50 \mathrm{~nm}$ was spin-coated on the quartz substrate in advance. It functions as an adhesive layer to ensure the firm attachment of the photoresist grating. The detailed fabrication procedure can be found in Supporting Information. The width $\left(W_{2}\right)$ of each gold stripe is slightly larger than the width $\left(W_{1}\right)$ of the top of the slanted photoresist stripe as seen from the cross-sectional view scanning electron microscopy (SEM) image (Figure $3 \mathrm{~h}$ ). Figure $3 \mathrm{j}$ show the top-view SEM image of the dislocated double-layer metal grating. In this picture, two large grooves were etched by focused ion beam etcher (FIB, Zess AURIGA), and a finite structure with 7 periods is formed. The big grooves at the both ends of the finite structure were used to scatter and monitor the coupled SPP. To compare the difference between the dislocated double-layer metal grating and nondislocated double-layer metal grating we also fabricated nondislocated structures with nearly the same period and filling fraction (Figure 3g,i). The parameters of the fabricated dislocated structure are $H=490 \mathrm{~nm}, W_{1}=240 \mathrm{~nm}, W_{2}=290 \mathrm{~nm}, P=770$ $\mathrm{nm}, t=120 \mathrm{~nm}$, and $\Delta x=140 \mathrm{~nm}$; the parameters for nondislocated structure are the same except that $\Delta x=0 \mathrm{~nm}$.

All zeroth-order reflection spectra were measured on a UV/ visible/near-infrared spectrometer (Lambda 950, PerkinElmer) at normal incidence under p-polarization (indicated in the inset of Figure 1). The measured reflection spectra of both the periodic nondislocated double-layer metal grating and dislocated double-layer metal grating are shown in Figure $3 c, d$, respectively. A reflection dip at $895 \mathrm{~nm}$ appears only in the dislocated structure, which is responsible for the unidirectional mode. The spectra are in accordance with the simulation results in Figure 3e,f. Then the nondislocated structure and dislocated structure with 7 periods (Figure 3i,j) were observed in a homemade confocal optical microscope at the wavelength of $895 \mathrm{~nm}$. The numerical aperture of the objective lens (Olympus MPlanFLX100) is 0.9. The observed images were recorded by a semiconductor-cooled CCD camera, as shown in
Figure 3k,1. For the nondislocated structure, there exists a reflected spot and no side coupling for SPP. For the dislocated structure, there is a unidirectional coupling for SPP, and as a result we observe the scattered light spots at both sides of the reflected spot (red-dashed circle) in Figure 31. It is clear that the most of the SPP propagates to the right. Note that the reflected beam image for the dislocated grating shown in Figure 31 is significantly different from that for the nondislocated grating shown in Figure 3k due to the coupling of the incident beam to the unidirectional SPP. The ratio of the integrated scattered light intensity at the right side to that of the left side in the image (Figure 31) represents the contrast ratio. The coupling efficiency is calculated by the ratio of the integrated light intensity of the scattered light at the right side (Figure 31) to that of the light reflected by a silver mirror. We note that the incident laser is a pulse with a width of around $120 \mathrm{fs}$ and corresponds to a wavelength bandwidth of $10 \mathrm{~nm}$. With such a relatively broadband pulse, the measured contrast ratio and coupling efficiency were 43 and $36 \%$, respectively. Our structure therefore operates over a substantial bandwidth with high performance. This measured coupling efficiency is lower than that in the simulation results due to several experimental limitations; only a portion of the coupled light can be scattered out from the large grooves. Also, a focused Gaussian beam rather than a collimated one is used. With high performance, simple fabrication, and compact sizes, the proposed dislocated double-layer metal grating here can be a promising unidirectional coupler for SPPs in plasmonic devices.

In order to get insights into the process of unidirectional coupling, we examined infinitely periodic dislocated and nondislocated double-layer metal gratings by numerical simulation. Comparing the numerically calculated reflection spectra of these two structures at normal incidence, we see that the dislocated structure (Figure 3f) has an extra dip at the wavelength of $895 \mathrm{~nm}$ as compared to the nondislocated one (Figure 3e). This extra dip corresponds to the unidirectional mode. Also, the locations of the peaks and dips in the simulated spectra are in good agreement with those of experimentally measured reflection spectra shown in Figure 3c,d, which shows that the infinitely periodic structures are useful models for understanding the actual finite structures.

Next, to facilitate analytic modeling we further simplify the infinitely periodic structure by replacing the photoresist grating between the two metal grating layers with a uniform effective medium as shown in Figure 4a. The refractive index of the effective medium is $n_{\text {eff }}=1.21$, as obtained from the volumeweighted average between the dielectric constants of air and photoresist. For this simplified structure, we simulated the absorptivity as a function of the thickness $H^{\prime}$ of the effective medium and vacuum wavelength as shown in Figure $4 \mathrm{~b}$ for the dislocated structure.

The several strong absorption lines displayed in Figure $4 \mathrm{~b}$ can be easily explained by simple analytic theories. First, the absorption line around the wavelength of $1140 \mathrm{~nm}$, marked by a dashed line, is due to the excitation of the lattice plasmon polariton at the interface between the lower gold grating and PMMA layer underneath.

The remaining absorption lines are attributed to the excitation of guided modes formed between the two gold gratings. The condition for the guiding is ${ }^{44}$ 
(a)

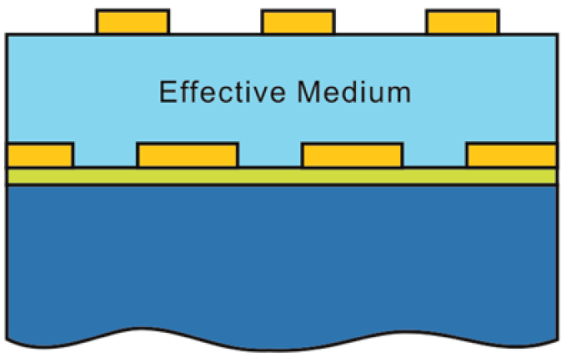

(b)

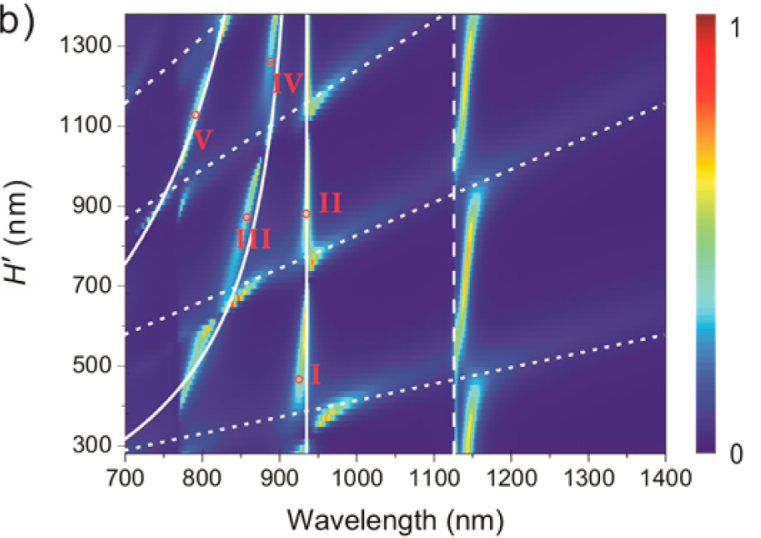

(c)

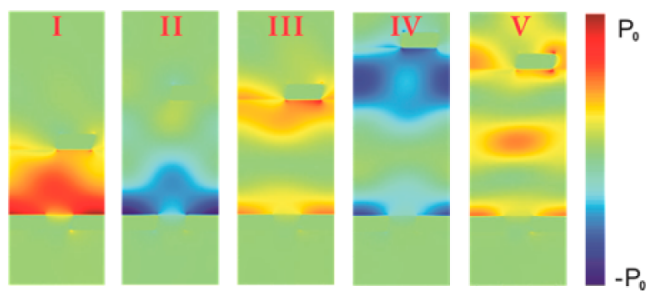

Figure 4. (a) A simplified structure with an effective medium uniformly filling the space between two metal grating layers; the thickness of this layer is $H^{\prime}$. (b) The simulated absorption spectra of simplified dislocated double-layer metal gratings $(\Delta x=140 \mathrm{~nm})$ with different thickness of the spacer between two gold gratings at normal incidence. Solid lines are calculated by eq 1 with $m=1$, dotted lines are calculated by eq 1 with $m=0$, and a vertical dashed line corresponds to the Wood's anomaly occurring at the interface between the lower gold grating and PMMA layer underneath. (c) The power flow density component $P_{x}$ (in the $x$-direction) distribution of dislocated structures with a few specific thicknesses and wavelengths marked by circles in (b). Red and blue colors represent the power flows to the right and left direction, respectively. The geometric parameters are $W_{1}=230 \mathrm{~nm}, W_{2}=270 \mathrm{~nm}, P=770 \mathrm{~nm}$, and $t=120$ nm.

$$
H^{\prime} \sqrt{\left(\frac{2 n_{\mathrm{eff}}}{\lambda_{0}}\right)^{2}-\left(\frac{2 m}{P}\right)^{2}}=n
$$

where $m \geq 0$ and $n \geq 0$ are integers, and $\lambda_{0}$ is the wavelength in vacuum. For $m=0$, the equation is simplified to $2 n_{\text {eff }} H^{\prime}=n \lambda_{0}$, which is indicated by the white dotted lines in Figure $4 \mathrm{~b}$. These absorption lines for $m=0$ occur in both the dislocated structures and nondislocated structures (The absorption spectra of nondislocated structure is shown in Figure S2 of Supporting Information). For $m=1$, eq 1 is indicated by the white solid lines. The absorption lines for $m=1$ exist in the dislocated double-layer metal gratings only, and they are in fact unidirectional modes, as can been seen from the $x$-component of power flow density $P_{x}$ (Figure 4c). It is interesting to note that the sign of $P_{x}$ changes every time as $H^{\prime}$ increases and crosses the dotted lines in Figure $4 \mathrm{~b}$ for a given wavelength. We also note that the case for $m=1, n=0$ (I, II in Figure $4 \mathrm{~b}$ ) corresponds to Wood's anomaly at the interfaces between the effective medium and gold gratings.

Below, we explain the unidirectionality with an analytic model. When a wave is normally incident on the upper gold grating, it is scattered into the effective medium by the periodic array of gold scatterers, as schematically shown in Figure 5. The

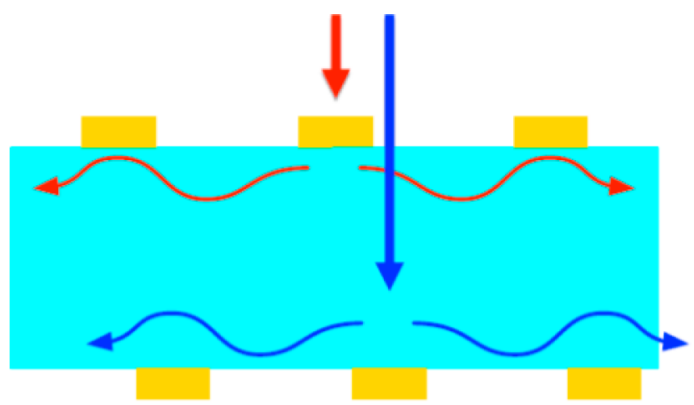

Figure 5. A schematic description of the interference model. The portions of a normally incident wave indicated by the red and blue vertical arrows are illuminated on the upper and lower gold gratings, respectively, and scattered into the effective medium to excite the horizontally guided waves. Because of the dislocation of the upper grating with respect to the lower grating, the guided waves excited at the upper and lower gold gratings can interfere differently depending on the propagation direction.

wave function generated by the scattering from the upper gold grating can be expressed as

$$
\psi_{\text {upper }}(x)=A \mathrm{e}^{-i \beta x}+A e^{+i \beta x}
$$

where $A$ is the complex amplitude and $\beta$ is the guide wavevector. The two terms on the right-hand side correspond to the components propagating in the $+x$ and $-x$ direction. Note that the complex amplitudes of the two components are the same due to the symmetry of the upper metal grating alone. The scattering by the lower gold grating generates a similar wave function but because of its dislocation with respect to the upper grating the wave function has the form

$$
\psi_{\text {lower }}(x)=B \mathrm{e}^{-i \beta(x+\Delta x)}+B e^{+i \beta(x+\Delta x)}
$$

The total wave function of the excited guided wave is the superposition of these wave functions

$$
\psi_{\text {total }}(x)=\left(A+B \mathrm{e}^{-i \beta \Delta x}\right) \mathrm{e}^{-i \beta x}+\left(A+B e^{+i \beta \Delta x}\right) e^{+i \beta x}
$$

The resulting wave function in eq 4 thus exhibits directiondependent constructive or destructive interference. For example, if $A=1, B=0.5 e^{+i \pi / 2}$, and $\Delta x=\pi / 2 \beta$, then $\psi_{\text {total }}(x)$ $=1.5 e^{-i \beta x}+0.5 e^{+i \beta x}$ whose net power flows in the $+x$ direction. Therefore, the dislocation of the two gold gratings plays a key role for the unidirectional coupling for surface plasmon. The optimal value of $\Delta x$ for efficient unidirectional coupling is empirically determined to be between $P / 8$ and $3 P / 8$ (see Supporting Information for details).

In conclusion, we have proposed theoretically and demonstrated experimentally a compact dislocated doublelayer metal grating unidirectional coupler for SPPs. Our 
structure is the first one that forms a unidirectional coupler by tuning the spacing and dislocation between two gratings. The origin of the unidirectional coupling in the structure is the dislocation-induced interference of the diffracted light from upper and lower gold gratings. The effects of the distance and dislocation between the two gold gratings on the strength of the unidirectional coupling were analyzed by a simple analytic model in order to optimize the structure. Especially for the lowest unidirectional coupling mode for SPPs, the structure parameters can be analytically determined by this simple model and dispersion equation. The simulated contrast ratio and unidirectional coupling efficiency of the optimized structure with 7 periods are 78 and 49\%, respectively. We then developed a high throughput, simple and cost-effective method to prepare the structure via tilted two-beam interference lithography and shadow deposition of gold. The fabrication method is scalable and also compatible with the modern batch fabrication process, which is extremely important for applications in integrated optical circuits. The experimentally measured contrast ratio and unidirectional coupling efficiency are 43 and 36\%, respectively, under normal illumination by a focused Gaussian beam. We believe that this structure is applicable to a variety of plasmonic devices requiring efficient unidirectional coupling of light into SPPs.

\section{ASSOCIATED CONTENT}

\section{S Supporting Information}

The detailed fabrication procedure of dislocated double-layer metal gratings. Derivation of eq 1 . The absorption spectra of nondislocated double-layer metal gratings. The effect of dislocation on the performance of the unidirectional coupling for SPPs. This material is available free of charge via the Internet at http://pubs.acs.org.

\section{AUTHOR INFORMATION}

\section{Corresponding Authors}

*E-mail: (C.J.) jinchjun@mail.sysu.edu.cn.

*E-mail: (S.F.) shanhui@stanford.edu.

\section{Author Contributions}

${ }^{\S}$ T.L., Y.S., and W.S. contributed equally.

\section{Notes}

The authors declare no competing financial interest.

\section{ACKNOWLEDGMENTS}

The authors acknowledge the financial support from the Chinese National Key Basic Research Special Fund (2010CB923201), the National Natural Science foundation of China (11374376, 11174374), the Key project of DEGP (No. 2012CXZD0001), and Innovative Talents Training Program for Doctoral Students of Sun Yat-sen University. W.S. and S.F. acknowledge the support of an AFOSR MURI program. (Grant FA9550-12-1-0024).

\section{REFERENCES}

(1) Barnes, W. L.; Dereux, A.; Ebbesen, T. W. Nature 2003, 424, 824-830.

(2) Maier, S. A.; Kik, P. G.; Atwater, H. A.; Meltzer, S.; Harel, E.; Koel, B. E.; Requicha, A. A. Nat. Mater. 2003, 2, 229-232.

(3) Briggs, R. M.; Grandidier, J.; Burgos, S. P.; Feigenbaum, E.; Atwater, H. A. Nano Lett. 2010, 10, 4851-4857.

(4) Delacour, C.; Blaize, S.; Grosse, P.; Fedeli, J. M.; Bruyant, A.; Salas-Montiel, R.; Lerondel, G.; Chelnokov, A. Nano Lett. 2010, 10, 2922-2926.
(5) Søndergaard, T.; Bozhevolnyi, S. I.; Boltasseva, A. Phys. Rev. B 2006, 73, 045320.

(6) Wang, B.; Aigouy, L.; Bourhis, E.; Gierak, J.; Hugonin, J. P.; Lalanne, P. Appl. Phys. Lett. 2009, 94, 011114.

(7) Lee, S. Y.; Lee, I. M.; Park, J.; Oh, S.; Lee, W.; Kim, K. Y.; Lee, B. Phys. Rev. Lett. 2012, 108, 213907.

(8) Wang, Y.; Wang, L. L.; Liu, J. Q.; Zhai, X.; Wang, L.; Xiang, D.; Xiang, Q.; Meng, B. Opt. Commun. 2010, 283, 1777-1779.

(9) Rodríguez-Fortuño, F. J.; Marino, G.; Ginzburg, P.; O’Connor, D.; Martínez, A.; Wurtz, G. A.; Zayats, A. V. Science 2013, 340, 328330.

(10) Lin, J.; Mueller, J. B.; Wang, Q.; Yuan, G.; Antoniou, N.; Yuan, X. C.; Capasso, F. Science 2013, 340, 331-334.

(11) He, M. D.; Liu, J. Q.; Wang, K. J.; Wang, X. J.; Gong, Z. Q. Opt. Commun. 2012, 285, 4588-4592.

(12) Gan, Q.; Bartoli, F. J. Opt. Lett. 2010, 35, 4181-4183.

(13) Lu, F.; Li, G.; Xiao, F.; Xu, A. IEEE Photon. J. 2012, 4, 17441751.

(14) Gan, Q.; Guo, B.; Song, G.; Chen, L.; Fu, Z.; Ding, Y. J.; Bartoli, F. J. Appl. Phys. Lett. 2007, 90, 161130.

(15) Wang, Y.; Zhang, X.; Tang, H.; Yang, K.; Wang, Y.; Song, Y.; Wei, T. H.; Wang, C. H. Opt. Express 2009, 17, 20457-20464.

(16) Li, D.; Zhang, D. H.; Yan, C.; Li, T.; Wang, Y.; Xu, Z.; Wang, J.; Qin, F. Opt. Express 2013, 21, 5949-5956.

(17) Choi, S. B.; Park, D. J.; Jeong, Y. K.; Yun, Y. C.; Jeong, M. S.; Byeon, C. C.; Kang, J. H.; Park, Q. -Han.; Kim, D. S. Appl. Phys. Lett. 2009, 94, 063115.

(18) Chen, J.; Li, Z.; Yue, S.; Gong, Q. Appl. Phys. Lett. 2010, 97, 041113.

(19) López-Tejeira, F.; Rodrigo, S. G.; Martín-Moreno, L.; GarcíaVidal, F. J.; Devaux, E.; Ebbesen, T. W.; Krenn, J. R.; Radko, I. P.; Bozhevolnyi, S. I.; González, M. U.; Weeber, J. C.; Dereux, A. Nat. Phys. 2007, 3, 324-328.

(20) Xu, T.; Zhao, Y.; Gan, D.; Wang, C.; Du, C.; Luo, X. Appl. Phys. Lett. 2008, 92, 101501.

(21) Liu, J. S.; Pala, R. A.; Afshinmanesh, F.; Cai, W.; Brongersma, M. L. Nat. Commun. 2011, 2, 525.

(22) Liu, Y.; Palomba, S.; Park, Y.; Zentgraf, T.; Yin, X.; Zhang, X. Nano Lett. 2012, 12, 4853-4858.

(23) Lerosey, G.; Pile, D. F. P.; Matheu, P.; Bartal, G.; Zhang, X. Nano Lett. 2009, 9, 327-331.

(24) Verslegers, L.; Yu, Z.; Catrysse, P. B.; Fan, S. J. Opt. Soc. Am. B 2010, 27, 1947-1956.

(25) Bonod, N.; Popov, E.; Li, L.; Chernov, B. Opt. Express 2007, 15, 11427-11432.

(26) Bai, B.; Meng, X.; Laukkanen, J.; Sfez, T.; Yu, L.; Nakagawa, W.; Herzig, H. P.; Li, L.; Turunen, J. Phys. Rev. B 2009, 80, 035407.

(27) Roszkiewicz, A.; Nasalski, W. J. Phys. B: At. Mol. Opt. Phys. 2010, 43, 185401

(28) Bouillard, J. S.; Vilain, S.; Dickson, W.; Wurtz, G. A.; Zayats, A. V. Sci. Rep. 2012, 2, 829.

(29) Huang, X.; Brongersma, M. L. Nano Lett. 2013, 13, 5420-5424.

(30) Huang, X.; Brongersma, M. L. Phys. Rev. B 2011, 84, 245120.

(31) Lu, J.; Petre, C.; Yablonovitch, E.; Conway, J. J. Opt. Soc. Am. B 2007, 24, 2268-2272.

(32) Baron, A.; Devaux, E.; Rodier, J. C.; Hugonin, J. P.; Rousseau, E.; Genet, C.; Ebbesen, T. W.; Lalanne, P. Nano Lett. 2011, 11, 42074212.

(33) Adams, J. L.; Botten, L. C. J. Opt. (Paris) 1979, 10, 109-117.

(34) Taubert, R; Ameling, R.; Weiss, T.; Christ, A.; Giessen, H. Nano Lett. 2011, 11, 4421-4424.

(35) Kappel, C.; Selle, A.; Bader, M. A.; Marowsky, G. J. Opt. Soc. Am. B 2004, 21, 1127-1136.

(36) Wang, L.; Zhang, Z. M. J. Opt. Soc. Am. B 2010, 27, 2595-2604.

(37) Zhou, L.; Wang, Q. J.; Wu, S.; Huang, W. X.; Huang, C. P.; Zhu, Y. Y. J. Opt. Soc. Am. B 2011, 28, 587-591.

(38) Fong, K. Y.; Hui, P. M. Appl. Phys. Lett. 2006, 89, 091101. 
(39) Chan, H. B.; Marcet, Z.; Woo, K.; Tannerb, D. B.; Carr, D. W.; Bower, J. E.; Cirelli, R. A.; Ferry, E.; Klemens, F.; Miner, J.; Pai, C. S.; Taylor, J. A. Opt. Lett. 2006, 31, 516-518.

(40) Cheng, C.; Chen, J.; Shi, D. J.; Wu, Q. Y.; Ren, F. F.; Xu, J.; Fan, Y. X.; Ding, J.; Wang, H. T. Phys. Rev. B 2008, 78, 075406.

(41) Marcet, Z.; Paster, J. W.; Carr, D. W.; Bower, J. E.; Cirelli, R. A.; Klemens, F.; Mansfield, W. M.; Miner, J. F.; Pai, C. S.; Chan, H. B. Opt. Lett. 2008, 33, 1410-1412.

(42) Estruch, T.; Jaeck, J.; Pardo, F.; Derelle, S.; Primot, J.; Pelouard, J. L.; Haidar, R. Opt. Lett. 2011, 36, 3160-3162.

(43) Johnson, P. B.; Christy, R. W. Phys. Rev. B 1972, 6, 4370.

(44) The detailed derivation can be found in Supporting Information. 